\title{
Development of Methods and Algorithms for Spectral Data Analysis for Vibroacoustic Diagnostics of Diesel-Generator Sets at NPPs
}

\section{Raspopov D. A. and Belousov P. A.}

Obninsk Institute for Nuclear Power Engineering of the National Research Nuclear University MEPhl, Studgorodok 1, Obninsk, Kaluga region, 249040, Russia

\section{Abstract}

In this article, the main methods and algorithms for spectral data analysis for vibroacoustic diagnostics of diesel-generator sets at nuclear power plants are considered. To collect the diagnostic data, an experimental setup was developed, thanks to which the sound signals of the diesel generator were obtained under various operating conditions. The recording and processing of signals was carried out using

Corresponding Author:

Raspopov D. A.

raspopovdmitriyalexeevich@ gmail.com

Received: 23 December 2017

Accepted: 15 January 2018

Published: 21 February 2018

Publishing services provided by Knowledge $\mathrm{E}$

(c) Raspopov D. A. and Belousov P. A.. This article is distributed under the terms of the

Commons Attribution License, which permits unrestricted use and redistribution provided that the original author and source are credited.

Selection and Peer-review under the responsibility of the AtomFuture Conference Committee.

\section{G OPEN ACCESS} the application package and MATLAB programming language. The article describes the application of correlation and spectral analysis for data processing and analysis. Also, the authors apply regression analysis to find the dependence of the speed of the diesel engine on the frequency of acoustic oscillations. The prediction of the number of revolutions from the frequency of sound vibrations makes it possible in the future to build a more accurate mathematical model of engine operation, and also to find diagnostic features for detecting malfunctions and anomalies in the operation of a diesel generator.

Keywords: NPPs, diesel generator sets, vibroacoustic diagnostics, spectral analysis, acoustic signals, MATLAB.

\section{Introduction}

Diesel generator sets (DGS) - a device that converts the mechanical energy of rotation of the crankshaft of a diesel engine into electrical energy generated by the alternator. The main advantages of the DGS are: high power ratings (up to 10-15 MW), versatility of application (can be both main and backup and emergency source), long time of trouble-free operation (enough to fill the fuel tank in time), much higher safety than gasoline and gas analogs (not so explosive), high efficiency, is 30-40\% and the average cost of fuel. The drawbacks of DGS include their relatively high cost, high noise level, 
inability to work at idle and poor tolerance of low temperatures. Thus, the DGS is the most cost-effective, reliable and efficient source for emergency power supply of NPPs.

The main purpose of the DGS at the NPPs is to maintain the safety of the power unit in the event of failure of the main power supply sources. Control over technical condition of diesel generator sets is a necessary condition to ensure the reliable functioning of safety systems of nuclear power plants, where vibroacoustic diagnostics is an important method used to control the technical condition of the DGS.

The topic chosen is important because the DGS at nuclear power plants during emergency situations involving loss of electrical power provide emergency power supply for consumers of the second category, having in its composition important safety system whose main function is to limit the consequences of possible accidents as explained in [1]. If the DGS is not launched in a timely manner, an accident related to de-energization can lead to a major catastrophe and irreparable consequences, for example, as at the Fukushima-1 NPP.

Improvement and development of methods for processing and analyzing diagnostic data for vibroacoustic diagnostics is the most important task for ensuring the highquality and reliable operation of the DGS at NPPs. Complex diagnostics of the dieselgenerator set includes several stages:

- general inspection (check of completeness of equipment, levels, density of process fluids, detection of visible leaks, condition of nozzles, condition and tension of drive belts, etc.);

- starting the engine, checking idling (uniformity of operation, noise level, color and odor of exhaust)

- diagnostics of the electrical part of the DGU (checking electrical connections, insulation resistance of wires, winding of the rotor and stator,);

- load testing (step-by-step check of the DGU in various power modes from 10\% to $110 \%$ of the rated power, load reset / load.

The reliable operation of the diesel generator set is provided primarily by its engine, which is why it is important to carred out a timely diagnosis of the diesel engine. Considering the high requirements for reliability of the DGS, it is necessary to solve the following tasks:

- identification of defects in the early stages of development;

- determination of the state trend;

- state forecasting. 
To solve these problems at nuclear power plants, vibroacoustic diagnostics methods are used. Vibroacousctic diagnostics is a diagnosis based on the measurement and analysis of noise and vibration, the levels of which vary depending on the mode of operation and the technical state of the systems and mechanisms of the DGS as explained in [2]. For vibratory noise diagnostics of the DGS, oscillatory processes of the elastic medium that arising from the operation of the mechanisms that are part of the installation.

\section{Materials and methods}

The aim of the work was to develop methods and algorithms for the analysis of spectral data for the diagnosis of diesel generators at NPPs. For the development of methods and algorithms for spectral analysis of data for vibroacoustic diagnostics, an experiment was conducted, the purpose of which was sound recording to a computer using acoustic sensors (microphones) with a frequency range of $4 \mathrm{~Hz}-20 \mathrm{kHz}$ of engine noise in two different modes (at high revs and at idling speed). An experimental setup was developed, consisting of a monitoring object - a DGU, four laptops and acoustic sensors. As an ADC, an external 3D C-Media CM108 sound card was used. To process the data received, the MATLAB programming language was used. The object of the control was taken as stationary diesel driven generator Champion DG $12 \mathrm{E}$ nominal power of $10 \mathrm{~kW}$ with a synchronous current generator and a four-stroke diesel engine. The block diagram of the experimental setup is shown in Figure 1, in which the digits 1,2,3,4 represent microphones, and 5,6,7,8 - laptops with external sound cards.

MATLAB is a high-level language and an interactive environment for programming, numerical calculations and visualization of results. Using MATLAB, can analyze data, develop algorithms, create models and applications. In MATLAB an important role is assigned to specialized groups of programs called toolboxes. For digital processing of signals, images and data in MATLAB, the following types of packages were used: DSP Toolbox, Image Processing Toolbox, Wavelet Toolbox, Communication Toolbox and Filter Design Toolbox. These sets of functions allow solving a wide range of tasks for processing signals, images, designing digital filters and communication systems.

For the processing and synchronization of signals from acoustic sensors, correlation analysis was used, which makes it possible, if necessary, to estimate the temporal properties of a signal without using spectral analysis, for example, to estimate the rate of change or duration of a signal, and temporarily correlate one signal with another. The mutual correlation function (MCF) determines the time coupling of two signals 


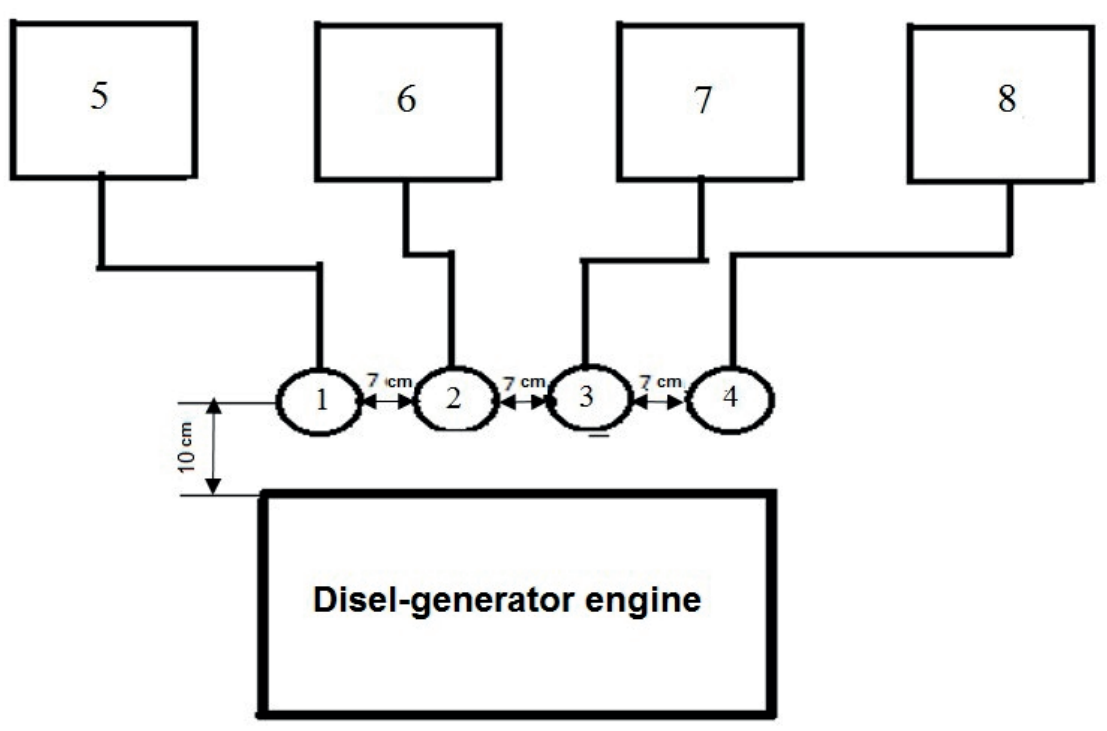

Figure 1: Diagram of the experimental setup.

in time. The autocorrelation function (ACF) shows the statistical relationship of one random variable over time.

Spectral analysis consists of decomposing a signal into its frequency or spectral components and evaluating or measuring their spectral characteristics. The mathematical basis of the spectral analysis of signals is the Fourier transform. The main purpose of the Fourier transform is to isolate the frequencies of the regular components of the signal, noisy with interference. A direct Fourier transform takes a description of the signal from the time domain to the frequency domain. Most often for spectral analysis, a fast Fourier transform (FFT) is used, by means of which the signal can be decomposed into its component oscillations of different frequency and amplitude. The windowed Fourier transform, unlike the usual one, is already a function of time, frequency, and amplitude. That is, it allows to obtain a characteristic of frequency distribution of a signal with amplitude in time.

\section{Results}

After carrying out the experiment it was possible to obtain 2 groups of data, in which there by were 4 signals from each microphone. With the help of the MATLAB software, temporary implementations of signals were made while the engine was running at idle and increased rpm. Figure 2 shows examples of temporal realizations of signals from acoustic sensors obtained when the engine is running at high speed. The graphs show 


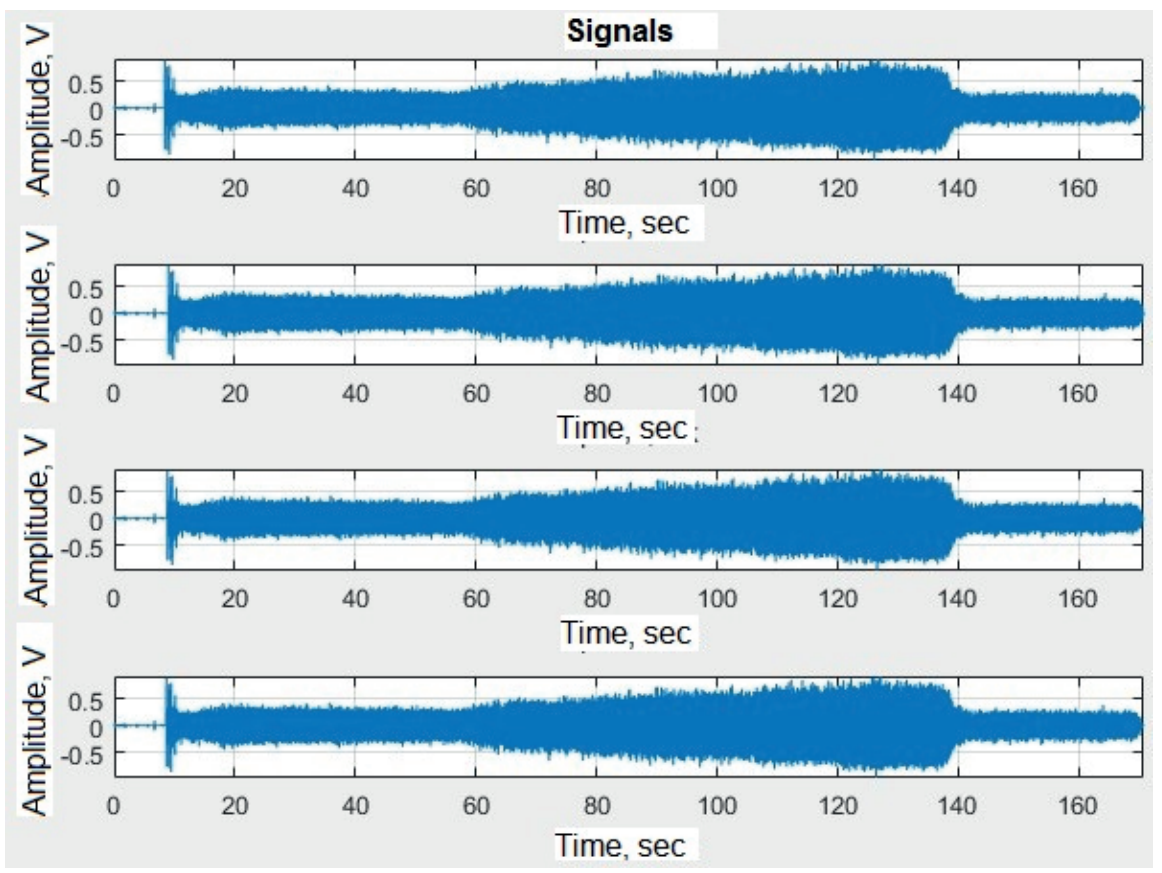

Figure 2: Time realization of signals from acoustic sensors.

that there is a slight delay among the signals. In order to remove it, we used methods of correlation analysis of the obtained data.

Correlation analysis was used to process and synchronize signals from acoustic sensors. Using the construction of a mutual correlation function, the values of the time delays between the signals, which are shown in Figure 3, were found.

Knowing the values of these delays, it was possible to synchronize the signals and again build a mutual correlation function already for the synchronized signals, which is shown in Figure 4.

After the synchronization and correlation analysis of signals from acoustic sensors, their spectral analysis was carried out using the techniques described in the book [3]. With the help of the Fourier transform in MATLAB, the spectra of acoustic signals for each mode of operation of the engine, shown in Figure 5, were plotted. The graph on the left shows the spectrum of the signal power received when the engine is running at constant revolutions, and on the right at elevated ones.

The spectra show how the power of the signal is distributed as a function of frequencies, that is, the power, which falls on a single frequency interval, is seen. It can also be said that at higher speeds, frequencies shift and the appearance of new signal powers.

The main task was to find the dependence of the number of revolutions on the frequency of acoustic oscillations. As a result of plotting this dependence, its linearity 


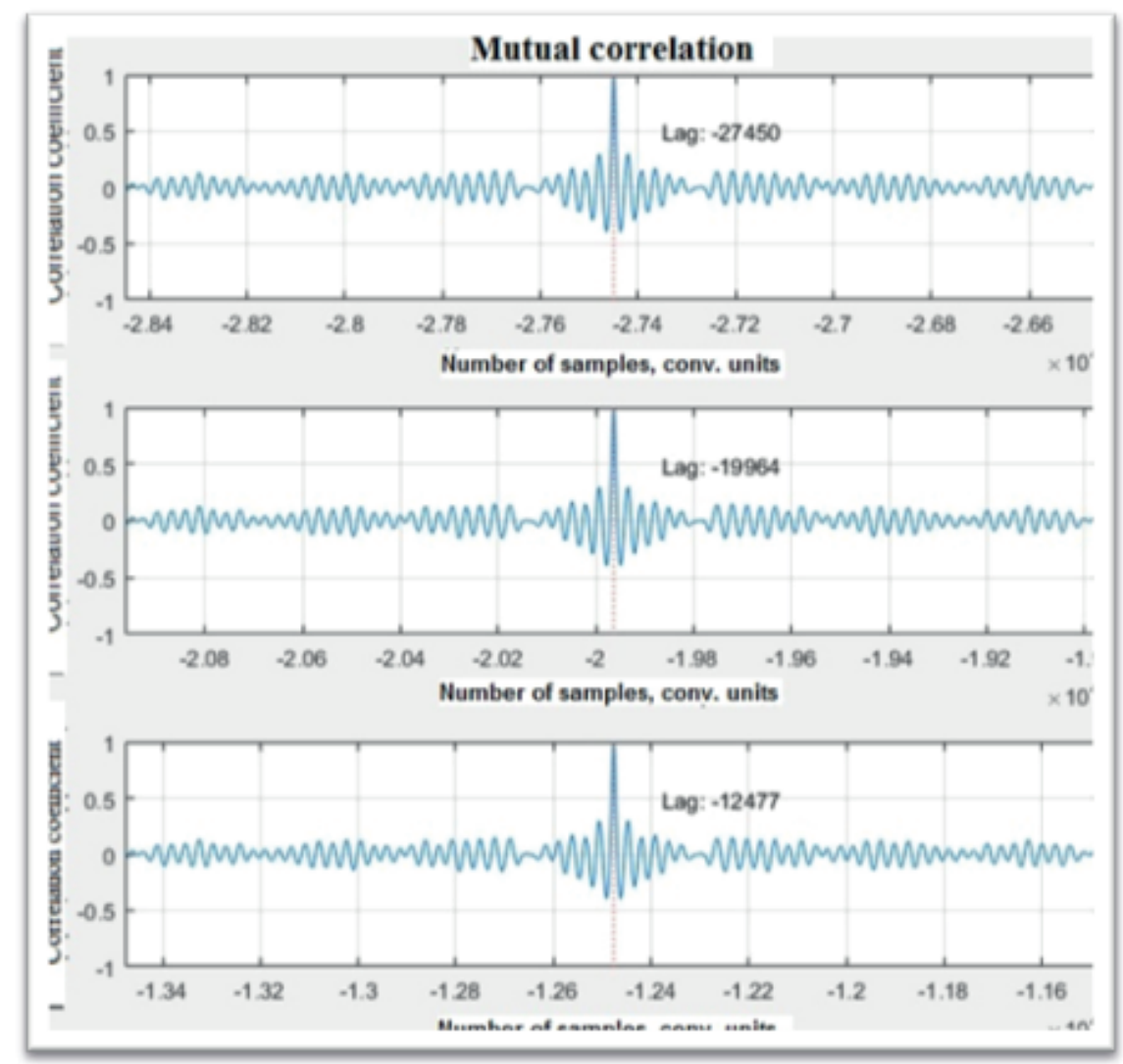

Figure 3: Mutual correlation function of signals from acoustic sensors.

was determined by the method of least quadarates. This dependence was found by isolating the frequencies at which the signal power was maximal, as well as observing the number of revolutions from measurements of the tachometer as a result of the experiment. As a result of plotting the graph of such a dependence, its linearity over the least squares was revealed. The graph of the dependence of the number of revolutions on the frequency of acoustic oscillations is shown in Figure 6.

Figure 6 shows that as the number of revolutions increases, the frequencies will shift and the line will continue. But there was not enough time for a full and error-free implementation of this method of diagnosis. Errors are possible because of the manual determination of the number of revolutions. Using the autocorrelation function, you can remove this error. In the future, it is planned to build an error-free dependence of the number of revolutions on the frequency of sound vibrations with the possibility of predicting them, and also to form a diagnostic feature and further develop an instrument based on the calculation of this dependence and the prediction of the state. 


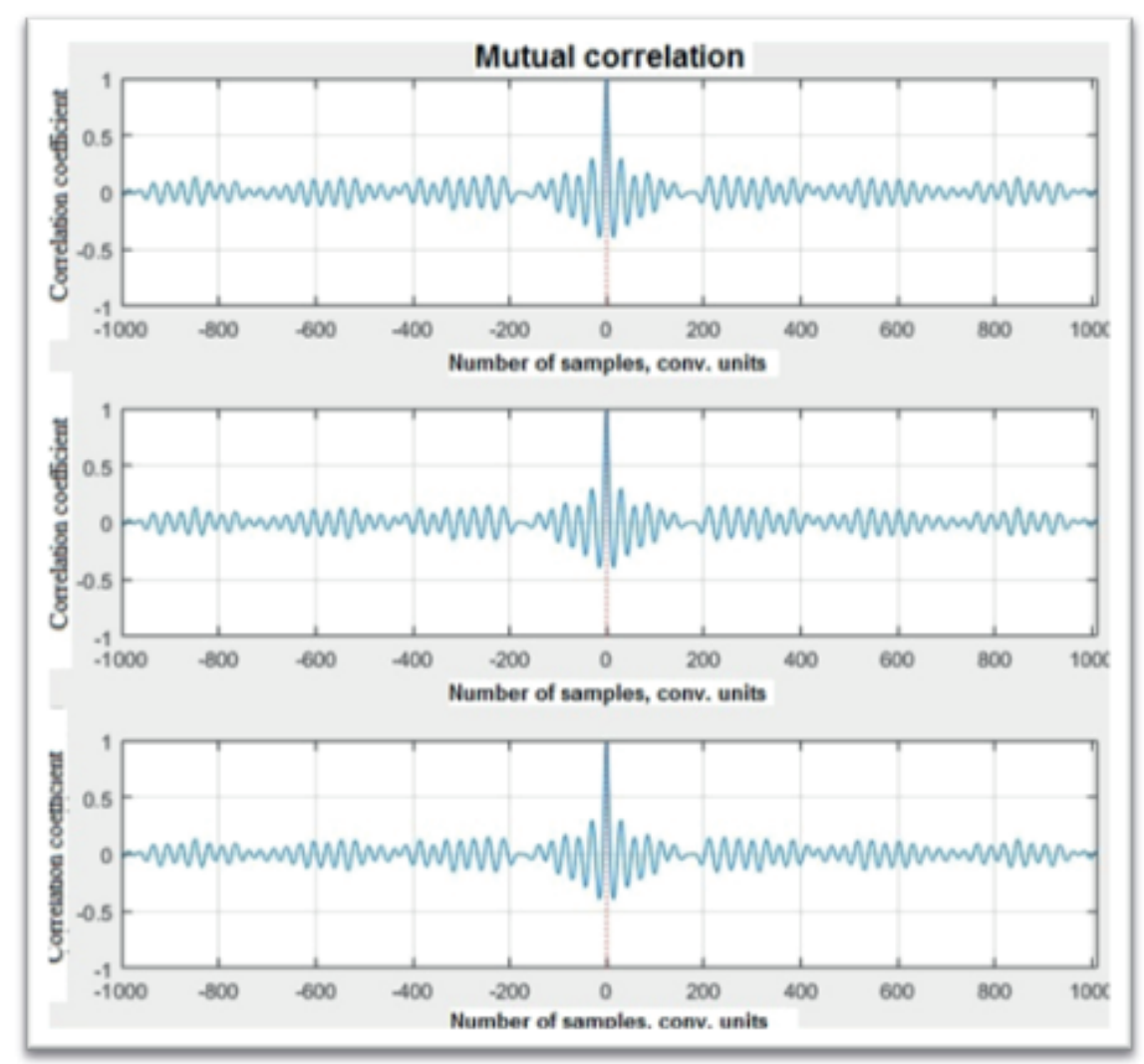

Figure 4: Mutual correlation function of synchronized signals from acoustic sensors.
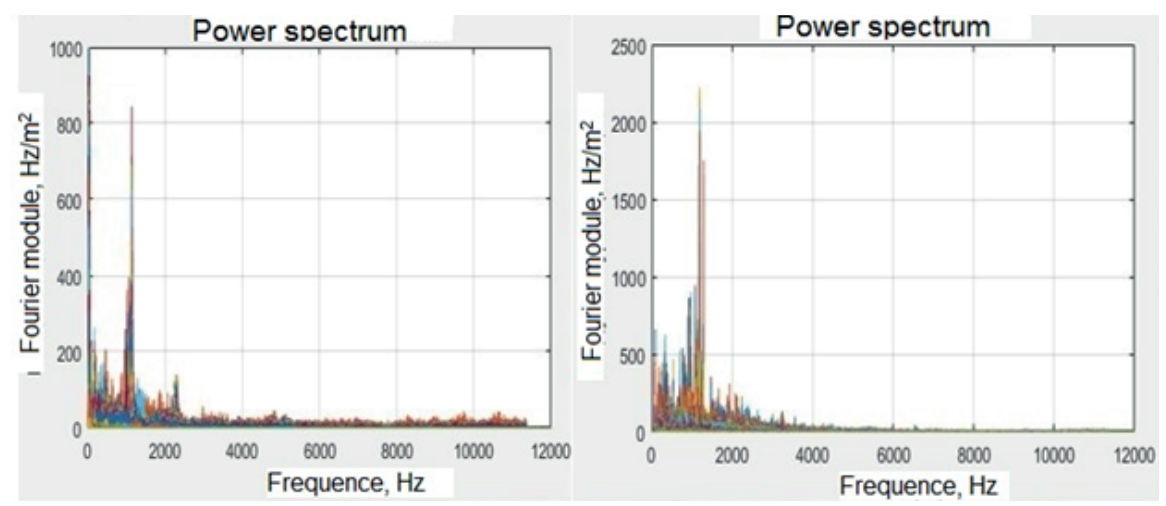

Figure 5: Spectra of signal power for two modes of operation of the DGS.

\section{Conclusion}

The prospect of this development is the possibility of predicting the number of revolutions from the frequency of sound vibrations, while if the characteristics of the engine were changed, it would be possible to construct a diagnostic feature and further develop an instrument based on the calculation of this dependence and the prediction of the state. Also in the future, it is planned to create a hardware and software complex for performing vibroacoustic diagnostics of the DGS at nuclear power plants. 


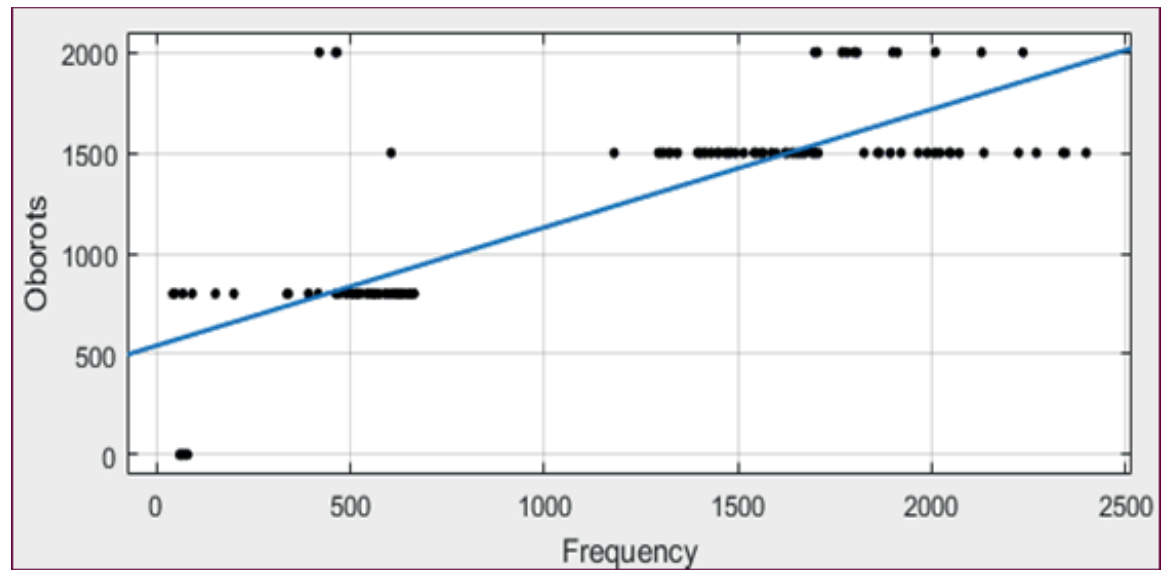

Figure 6: The dependence of the number of revolutions on the frequency of acoustic oscillations.

\section{References}

[1] Bukrinsky, A. M. Emergency transient processes at NPPs with VVER: productionpractical edition / A. M. Bukrinsky. - M.: Energoizdat, 1982. - 142 p.

[2] Genkin M. D., Sokolova A. G. Vibroacoustic diagnostics of machines and mechanisms. - M.: Mashinostroenie, 1987. -288C.

[3] Palano M. A., Romadanov V. M. Spectral transform in MATLAB. - SPb., 2007-160 p. 